doi: $10.13108 / 2021-13-1-119$

\title{
GENERALIZATION OF HADAMARD-TYPE TRAPEZOID INEQUALITIES FOR FRACTIONAL INTEGRAL OPERATORS
}

\author{
B. BAYRAKTAR, M. EMIN ÖZDEMIR
}

\begin{abstract}
The role of convexity theory in applied problems, especially in optimization problems, is well known. The integral Hermite-Hadamard inequality has a special place in this theory since it provides an upper bound for the mean value of a function. In solving applied problems from different fields of science and technology, along with the classical integro-differential calculus, fractional calculus plays an important role. A lot of research is devoted to obtaining an upper bound in the Hermite-Hadamard inequality using operators of fractional calculus.

The article formulates and proves the identity with the participation of the fractional integration operator. Based on this identity, new generalized Hadamard-type integral inequalities are obtained for functions for which the second derivatives are convex and take values at intermediate points of the integration interval. These results are obtained using the convexity property of a function and two classical integral inequalities, the HermiteHadamard integral inequality and its other form, the power mean inequality. It is shown that the upper limit of the absolute error of inequality decreases in approximately $n^{2}$ times, where $n$ is the number of intermediate points. In a particular case, the obtained estimates are consistent with known estimates in the literature. The results obtained in the article can be used in further researches in the integro-differential fractional calculus.
\end{abstract}

Keywords: convexity, Hermite-Hadamard inequality, Hölder inequality, power-mean inequality, Riemann-Liouville fractional Integrals.

Mathematics Subject Classification:26A51, 26D15

\section{INTRODUCTION}

The theory of integral inequalities is being actively developed since it is attracts a lot in interest. In recent decades, by using various methods, inequalities for different classes of convex functions were obtained. These classes of convex functions are based on the definition of the classical convexity of a function. This definition reads as follows (see, for instance, [1] and its references):

Definition 1. The function $f:[a, b] \rightarrow \mathbb{R}$ is said to be convex if we have

$$
f(t \xi+(1-t) \zeta) \leqslant t f(\xi)+(1-t) f(\zeta)
$$

for all $\xi, \zeta \in[a, b]$ and $t \in[0,1]$.

One of the most important inequalities in convex analysis is the Hermite-Hadamard integral inequality. This inequality is as follows [2]:

B. Bayraktar, M. Emin Özdemir, Generalization of Hadamard-type trapezoid inequalities for fractional integral operators.

(c) B. Bayraktar, M. Emin Özdemir. 2021.

Submitted April 1, 2020. 
Theorem 1.1. Let $f: I \subset \mathbb{R} \rightarrow \mathbb{R}$ be a convex function and let $a, b \in I$ with $a<b$. Then the following double inequality holds:

$$
f\left(\frac{a+b}{2}\right) \leqslant \frac{1}{b-a} \int_{a}^{b} f(x) d x \leqslant \frac{f(a)+f(b)}{2} .
$$

A natural extension of classical analysis is the integro-differential calculus. This theory plays a very important role in classical and applied mathematics( [3, 4] ). In mathematical modeling of complex systems and processes, the application of fractional calculus theory allows one to reflect adequately the properties of real systems and processes in models [4].

Butkovsky et al. [5], along with problems in the theory of fractional calculus, gave examples of real systems in which it is necessary to use that theory.

In the literature, there are various definitions of the fractional integral (see, for example, [6.7]), but the Riemann-Liouville definition is widely used in most applications of fractional calculus.

Definition 2. ([3]) Let $f \in L_{1}[a, b]$. The Riemann-Liouville integrals $J_{a^{+}}^{\alpha} f$ and $J_{b^{-}}^{\alpha} f$ in order $\alpha>0$ are defined respectively by

$$
J_{a^{+}}^{\alpha} f(\xi)=\frac{1}{\Gamma(\alpha)} \int_{a}^{\xi}(\xi-t)^{\alpha-1} f(t) d t, \quad \xi>a,
$$

and

$$
J_{b-}^{\alpha} f(\xi)=\frac{1}{\Gamma(\alpha)} \int_{\xi}^{b}(t-\xi)^{\alpha-1} f(t) d t, \quad \xi<b,
$$

where $\Gamma(\alpha)=\int_{0}^{\infty} e^{-u} u^{\alpha-1} d u$ is the well known Gamma function. Here for

$$
J_{a^{+}}^{0} f(\xi)=J_{b^{-}}^{0} f(\xi)=f(\xi)
$$

as $\alpha=0$ and

$$
J_{a^{+}}^{1} f(\xi)=J_{b^{-}}^{1} f(\xi)=\int_{a}^{b} f(\xi) d \xi
$$

as $\alpha=1$.

Two classical inequalities are used in the vast majority of studies on the theory of integral inequality. These are Hölder inequality and, its other form, the power mean inequality.

Theorem 1.2. (Hölder inequality [87) Let $p>1$ and $\frac{1}{p}+\frac{1}{q}=1$. If $f(\xi)$ and $g(\xi)$ are real functions defined on $[a, b]$ and if $|f|^{p},|g|^{q} \in L[a, b]$, then

$$
\int_{a}^{b}|f(\xi) g(\xi)| d \xi \leqslant\left(\int_{a}^{b}|f(\xi)|^{p} d \xi\right)^{\frac{1}{p}}\left(\int_{a}^{b}|g(\xi)|^{q} d \xi\right)^{\frac{1}{q}}
$$

with equality holds if and only if $A|f(\xi)|^{p}=B|g(\xi)|^{q}$ almost everywhere, where $A$ and $B$ are constants.

Theorem 1.3. (Power mean inequality [8]) Let $q \geqslant 1$ and $\frac{1}{p}+\frac{1}{q}=1$. If $f(\xi)$ and $g(\xi)$ are real functions defined on $[a, b]$ and if $|f|^{p},|g|^{q} \in L[a, b]$, then

$$
\int_{a}^{b}|f(\xi) g(\xi)| d \xi \leqslant\left(\int_{a}^{b}|f(\xi)| d \xi\right)^{1-\frac{1}{q}}\left(\int_{a}^{b}|f(\xi)||g(\xi)|^{q} d \xi\right)^{\frac{1}{q}}
$$


In recent years, many authors (see $[7-18]$ and references therein) studied Hermite-Hadamardtype inequalities for improvements and generalizations. In these papers, new inequalities for functions from various convexity classes were obtained.

In the studies [14 16], generalizations of an Hadamard-type integral inequality are given for functions with convex first derivatives taking values at intermediate points. In this study, by using the formulated identity, we obtain some new generalizations of Hadamard-type inequalities for functions such that the absolute values of the second derivatives are convex and take values at intermediate points of the interval. In addition, some applications to special means are given.

\section{MAIN RESULTS}

Let $n \in \mathbb{N}$ and $a, b \in \mathbb{R}$ and $a<b$. The interval $[a, b]$ with a uniform step $h=\frac{b-a}{n}$ is divided into $n$ subintervals: $[a, b]=\bigcup_{k=1}^{n}\left[\xi_{k-1}, \xi_{k}\right]$, where $\xi_{i}=a+i h, \quad i=0,1,2, \ldots, n$.

To avoid some repetitions, we use the following notation:

$A(.,$.$) is the arithmetic means of real numbers,$

$$
\begin{aligned}
y_{k} & =\frac{f\left(\xi_{k-1}\right)+f\left(\xi_{k}\right)}{2}, \\
U_{k} & =\frac{\alpha}{h}\left(J_{\xi_{k-1}^{+}}^{\alpha-1} f\left(\xi_{k}\right)+J_{\xi_{k}^{-}}^{\alpha-1} f\left(\xi_{k-1}\right)\right)-\left(J_{\xi_{k-1}^{+}}^{\alpha-2} f\left(\xi_{k}\right)+J_{\xi_{k}^{-}}^{\alpha-2} f\left(\xi_{k-1}\right)\right), \quad k=1,2, \ldots, n .
\end{aligned}
$$

The following theorem can be easily proved.

Theorem 2.1. Let $f:[a, b] \rightarrow \mathbb{R}$ be a differentiable function on $[a, b]$. If $f \in L[a, b]$, then for all $\alpha>0$ the following inequality holds:

$$
A\left(x_{1}, x_{2}, \ldots, x_{n}\right) \leqslant \frac{\Gamma(\alpha+1)}{2} A\left(s_{1}, s_{2}, \ldots, s_{n}\right) \leqslant A\left(y_{1}, y_{2}, \ldots, y_{n}\right),
$$

where

$$
x_{k}=f\left(\frac{\xi_{k-1}+\xi_{k}}{2}\right), \quad s_{k}=\frac{1}{\left(\xi_{k}-\xi_{k-1}\right)^{\alpha}}\left(J_{\xi_{k-1}^{+}}^{\alpha} f\left(\xi_{k}\right)+J_{\xi_{k}^{-}}^{\alpha} f\left(\xi_{k-1}\right)\right) .
$$

Remark 1. Inequality (2.1) can be treated as a corollary of Theorem 2 in [9]. For $n=1$, by (2.1), we get:

$$
f\left(\frac{a+b}{2}\right) \leqslant \frac{\Gamma(\alpha+1)}{2(b-a)^{\alpha}}\left[J_{a^{+}}^{\alpha} f(b)+J_{b^{-}}^{\alpha} f(a)\right] \leqslant \frac{f(a)+f(b)}{2} .
$$

Lemma 2.1. Let $f: I \subset \mathbb{R} \rightarrow \mathbb{R}$ be a twice differentiable mapping on $I^{\circ}$, where $I^{\circ}$ is the interior of $I$. If $f^{\prime \prime} \in L[a, b]$, where $a, b \in I$, then for all $\alpha>2$, the following identity holds

$$
\sum_{k=1}^{n} \frac{f\left(\xi_{k-1}\right)+f\left(\xi_{k}\right)}{2}-\frac{\Gamma(\alpha)}{2 h^{\alpha-2}} \sum_{k=1}^{n} U_{k}=\frac{h^{2}}{2} \sum_{k=1}^{n}\left(I_{1 k}+I_{2 k}\right),
$$

where

$$
\begin{aligned}
& I_{1 k}=\int_{0}^{1}\left(t^{\alpha-1}-t^{\alpha}\right) f^{\prime \prime}\left(t \xi_{k-1}+(1-t) \xi_{k}\right) d t, \\
& I_{2 k}=\int_{0}^{1}\left(t^{\alpha-1}-t^{\alpha}\right) f^{\prime \prime}\left((1-t) \xi_{k-1}+t \xi_{k}\right) d t .
\end{aligned}
$$


Proof. By taking into account that $h=\xi_{k}-\xi_{k-1}$ and integrating by parts twice integral $I_{1 k}$, we obtain

$$
\begin{aligned}
I_{1 k}= & \int_{0}^{1}\left(t^{\alpha-1}-t^{\alpha}\right) f^{\prime \prime}\left(t \xi_{k-1}+(1-t) \xi_{k}\right) d t \\
= & -\left.\frac{t^{\alpha-1}-t^{\alpha}}{h} f^{\prime}\left(t \xi_{k-1}+(1-t) \xi_{k}\right)\right|_{0} ^{1}+\frac{1}{h} \int_{0}^{1}\left((\alpha-1) t^{\alpha-2}-\alpha t^{\alpha-1}\right) f^{\prime}\left(t \xi_{k-1}+(1-t) \xi_{k}\right) d t \\
= & \frac{1}{h}\left(-\left.\frac{(\alpha-1) t^{\alpha-2}-\alpha t^{\alpha-1}}{h} f\left(t \xi_{k-1}+(1-t) \xi_{k}\right)\right|_{0} ^{1}\right. \\
& \left.+\frac{1}{h}(\alpha-1) \int_{0}^{1}\left((\alpha-2) t^{\alpha-3}-\alpha t^{\alpha-2}\right) f\left(t \xi_{k-1}+(1-t) \xi_{k}\right) d t\right) \\
= & \frac{f\left(\xi_{k-1}\right)}{h^{2}}+\frac{(\alpha-1)}{h^{2}}\left((\alpha-2) \int_{0}^{1} t^{\alpha-3} f\left(t \xi_{k-1}+(1-t) \xi_{k}\right) d t\right. \\
& \left.-\alpha \int_{0}^{1} t^{\alpha-2} f\left(t \xi_{k-1}+(1-t) \xi_{k}\right) d t\right) .
\end{aligned}
$$

In the obtained integrals, we change the variables $t \xi_{k-1}+(1-t) \xi_{k}=x$ and by taking into account the properties of the gamma function $(s \Gamma(s)=\Gamma(s+1))$, we obtain

$$
\begin{aligned}
I_{1 k} & =\frac{f\left(\xi_{k-1}\right)}{h^{2}}+\frac{(\alpha-1)}{h^{\alpha}}\left((\alpha-2) \int_{\xi_{k-1}}^{\xi_{k}}\left(\xi_{k}-x\right)^{\alpha-3} f(x) d x-\frac{\alpha}{h} \int_{\xi_{k-1}}^{\xi_{k}}\left(\xi_{k}-x\right)^{\alpha-2} f(x) d x\right) \\
& =\frac{f\left(\xi_{k-1}\right)}{h^{2}}+\frac{\Gamma(\alpha)}{h^{\alpha}}\left(J_{\xi_{k-1}^{+}}^{\alpha-2} f\left(\xi_{k}\right)-\frac{\alpha}{h} J_{\xi_{k-1}^{+}}^{\alpha-1} f\left(\xi_{k}\right)\right) .
\end{aligned}
$$

Similarly, for the other integral

$$
I_{2 k}=\frac{f\left(\xi_{k}\right)}{h^{2}}+\frac{\Gamma(\alpha)}{h^{\alpha}}\left(J_{\xi_{k}^{-}}^{\alpha-2} f\left(\xi_{k-1}\right)-\frac{\alpha}{h} J_{\xi_{k}^{-}}^{\alpha-1} f\left(\xi_{k-1}\right)\right) .
$$

By summing these identities, we obtain

or

$$
\begin{aligned}
I_{1 k}+I_{2 k}= & \frac{f\left(\xi_{k-1}\right)+f\left(\xi_{k}\right)}{h^{2}}-\frac{\Gamma(\alpha)}{h^{\alpha}}\left(\frac{\alpha}{h}\left(J_{\xi_{k-1}^{+}}^{\alpha-1} f\left(\xi_{k}\right)+J_{\xi_{k}^{-}}^{\alpha-1} f\left(\xi_{k-1}\right)\right)\right. \\
& \left.-\left(J_{\xi_{k-1}^{+}}^{\alpha-2} f\left(\xi_{k}\right)+J_{\xi_{k}^{-}}^{\alpha-2} f\left(\xi_{k-1}\right)\right)\right)
\end{aligned}
$$

$$
I_{1 k}+I_{2 k}=\frac{f\left(\xi_{k-1}\right)+f\left(\xi_{k}\right)}{h^{2}}-\frac{\Gamma(\alpha)}{h^{\alpha}} \cdot U_{k}
$$

By multiplying both sides of the equality 2.3 by the expression $\frac{h^{2}}{2}$ and taking the sum over $k$, we obtain (2.2). This completes the proof.

Theorem 2.2. Let $f: I \subset \mathbb{R} \rightarrow \mathbb{R}$ be twice differentiable function on $I^{\circ}$. If $f^{\prime \prime} \in L[a, b]$, where $a, b \in I$ and $\left|f^{\prime \prime}\right|$ is a convex function, then for all $\alpha>2$ the following inequality holds:

$$
\left|\sum_{k=1}^{n} \frac{f\left(\xi_{k-1}\right)+f\left(\xi_{k}\right)}{2}-\frac{\Gamma(\alpha)}{2 h^{\alpha-2}} \sum_{k=1}^{n} U_{k}\right| \leqslant \frac{h^{2}}{2 \alpha(\alpha+1)} \sum_{k=1}^{n}\left(\left|f^{\prime \prime}\left(\xi_{k-1}\right)\right|+\left|f^{\prime \prime}\left(\xi_{k}\right)\right|\right) .
$$


Proof. By Lemma 2.1 and the triangle inequality, we obtain

$$
\left|\sum_{k=1}^{n} \frac{f\left(\xi_{k-1}\right)+f\left(\xi_{k}\right)}{2}-\frac{\Gamma(\alpha)}{2 h^{\alpha-2}} \sum_{k=1}^{n} U_{k}\right| \leqslant \frac{h^{2}}{2} \sum_{k=1}^{n}\left(\left|I_{1 k}\right|+\left|I_{2 k}\right|\right) .
$$

Since $\left|f^{\prime \prime}\right|$ is convex with the account of inequality (1.1), we can write

$$
\begin{aligned}
\left|I_{1 k}\right| & =\left|\int_{0}^{1}\left(t^{\alpha-1}-t^{\alpha}\right) f^{\prime \prime}\left(t \xi_{k-1}+(1-t) \xi_{k}\right) d t\right| \\
& \leqslant\left|f^{\prime \prime}\left(\xi_{k-1}\right)\right| \int_{0}^{1} t\left(t^{\alpha-1}-t^{\alpha}\right) d t+\left|f^{\prime \prime}\left(\xi_{k}\right)\right| \int_{0}^{1}\left(t^{\alpha-1}-t^{\alpha}\right)(1-t) d t \\
& =\frac{1}{(\alpha+1)(\alpha+2)}\left|f^{\prime \prime}\left(\xi_{k-1}\right)\right|+\frac{2}{\alpha(\alpha+1)(\alpha+2)}\left|f^{\prime \prime}\left(\xi_{k}\right)\right| .
\end{aligned}
$$

Hence, for the integral $I_{1 k}$ we obtain

$$
\left|I_{1 k}\right| \leqslant \frac{1}{(\alpha+1)(\alpha+2)}\left(\left|f^{\prime \prime}\left(\xi_{k-1}\right)\right|+\frac{2}{\alpha}\left|f^{\prime \prime}\left(\xi_{k}\right)\right|\right)
$$

Similarly, for the second integral $\left|I_{2 k}\right|$ we can write

$$
\left|I_{2 k}\right| \leqslant \frac{1}{(\alpha+1)(\alpha+2)}\left(\frac{2}{\alpha}\left|f^{\prime \prime}\left(\xi_{k-1}\right)\right|+\left|f^{\prime \prime}\left(\xi_{k}\right)\right|\right)
$$

By adding the last two inequalities, we get

$$
\left|I_{1 k}\right|+\left|I_{2 k}\right| \leqslant \frac{1}{\alpha(\alpha+1)}\left(\left|f^{\prime \prime}\left(\xi_{k-1}\right)\right|+\left|f^{\prime \prime}\left(\xi_{k}\right)\right|\right) .
$$

By multiplying both sides of inequality 2.6 by the expression $\frac{h^{2}}{2}$ and taking into account inequality (2.5), we obtain (2.4). The proof is complete.

Corollary 1. If we choose $\alpha=2$, then from (2.4), we get

$$
\left|A\left(y_{1}, y_{2}, \ldots, y_{n}\right)-\frac{1}{b-a} \int_{a}^{b} f(x) d x\right| \leqslant \frac{(b-a)^{2}}{24 n^{2}} A\left(z_{1}, z_{2}, \ldots, z_{n}\right)
$$

where

$$
z_{k}=\left|f^{\prime \prime}\left(\xi_{k-1}\right)\right|+\left|f^{\prime \prime}\left(\xi_{k}\right)\right|, \quad k=1,2, \ldots, n .
$$


Proof. Since $h=\xi_{k}-\xi_{k-1}=\frac{b-a}{n}$ and $\alpha=2$, for the second expression under the absolute value in the right hand side of inequality (2.4) we have:

$$
\begin{aligned}
\frac{\Gamma(\alpha)}{2 h^{\alpha-2} \sum_{k=1}^{n} U_{k}} & =\frac{\Gamma(\alpha)}{2 h^{\alpha-2}} \sum_{k=1}^{n}\left(\frac{\alpha}{h}\left(J_{\xi_{k-1}^{+}}^{\alpha-1} f\left(\xi_{k}\right)+J_{\xi_{k}^{-}}^{\alpha-1} f\left(\xi_{k-1}\right)\right)-\left(J_{\xi_{k-1}^{+}}^{\alpha-2} f\left(\xi_{k}\right)+J_{\xi_{k}^{-}}^{\alpha-2} f\left(\xi_{k-1}\right)\right)\right) \\
& =\frac{1}{2}\left(\sum_{k=1}^{n} \frac{2}{h}\left(J_{\xi_{k-1}^{+}}^{1} f\left(\xi_{k}\right)+J_{\xi_{k}^{-}}^{1} f\left(\xi_{k-1}\right)\right)-\left(J_{\xi_{k-1}^{+}}^{0} f\left(\xi_{k}\right)+J_{\xi_{k}^{-}}^{0} f\left(\xi_{k-1}\right)\right)\right) \\
& =\frac{1}{2}\left(\frac{2 n}{b-a} \sum_{k=1}^{n}\left(\int_{\xi_{k-1}}^{\xi_{k}} f(x) d x+\int_{\xi_{k-1}}^{\xi_{k}} f(x) d x\right)-\sum_{k=1}^{n}\left(f\left(\xi_{k}\right)+f\left(\xi_{k-1}\right)\right)\right) \\
& =\frac{1}{2}\left(\frac{4 n}{b-a} \sum_{k=1}^{n}\left(\int_{\xi_{k-1}}^{\xi_{k}} f(x) d x\right)-\sum_{k=1}^{n}\left(f\left(\xi_{k}\right)+f\left(\xi_{k-1}\right)\right)\right) \\
& =\frac{1}{2}\left(\frac{4 n}{b-a} \int_{a}^{b} f(x) d x-\sum_{k=1}^{n}\left(f\left(\xi_{k}\right)+f\left(\xi_{k-1}\right)\right)\right) \\
& =\frac{2 n}{b-a} \int_{a}^{b} f(x) d x-\frac{1}{2} \sum_{k=1}^{n}\left(f\left(\xi_{k}\right)+f\left(\xi_{k-1}\right)\right) .
\end{aligned}
$$

Thus, we can rewrite inequality (2.4) as

$$
\begin{aligned}
& \left|\sum_{k=1}^{n} \frac{f\left(\xi_{k-1}\right)+f\left(\xi_{k}\right)}{2}-\frac{\Gamma(\alpha)}{2 h^{\alpha-2}} \sum_{k=1}^{n} U_{k}\right| \\
& =\left|\sum_{k=1}^{n} \frac{f\left(\xi_{k-1}\right)+f\left(\xi_{k}\right)}{2}-\left(\frac{2 n}{b-a} \int_{a}^{b} f(x) d x-\frac{1}{2} \sum_{k=1}^{n}\left(f\left(\xi_{k}\right)+f\left(\xi_{k-1}\right)\right)\right)\right| \\
& =\left|\sum_{k=1}^{n}\left(f\left(\xi_{k-1}\right)+f\left(\xi_{k}\right)\right)-\frac{2 n}{b-a} \int_{a}^{b} f(x) d x\right| \\
& \leqslant \frac{(b-a)^{2}}{12 n^{2}} \sum_{k=1}^{n}\left(\left|f^{\prime \prime}\left(\xi_{k-1}\right)\right|+\left|f^{\prime \prime}\left(\xi_{k}\right)\right|\right)
\end{aligned}
$$

or

$$
\left|\sum_{k=1}^{n} \frac{f\left(\xi_{k-1}\right)+f\left(\xi_{k}\right)}{2}-\frac{n}{b-a} \int_{a}^{b} f(x) d x\right| \leqslant \frac{(b-a)^{2}}{24 n^{2}} \sum_{k=1}^{n}\left(\left|f^{\prime \prime}\left(\xi_{k-1}\right)\right|+\left|f^{\prime \prime}\left(\xi_{k}\right)\right|\right) .
$$

By dividing both sides of the last inequality by $n$, we obtain (2.7). The proof is completed.

Corollary 2. If we choose $n=1$, by (2.7) we get

$$
\left|\frac{f(a)+f(b)}{2}-\frac{1}{b-a} \int_{a}^{b} f(x) d x\right| \leqslant \frac{(b-a)^{2}}{24}\left(\left|f^{\prime \prime}(a)\right|+\left|f^{\prime \prime}(b)\right|\right) .
$$

This inequality for convex functions was obtained by $M$. Sarlkaya and N. Aktan, see [18, Prop. 2], and in [11, Cor. 3.1]. 
Proposition 1. Let $\varepsilon \in(0,1)$ and $n \in N$. In order to satisfy the inequality,

$$
\left|A\left(y_{1}, y_{2}, \ldots, y_{n}\right)-\frac{1}{b-a} \int_{a}^{b} f(x) d x\right|<\varepsilon,
$$

it is sufficient to

$$
n>\left(\left|\frac{b-a}{2} \sqrt{\frac{\left\|f^{\prime \prime}\right\|}{3 \varepsilon}}\right|\right)
$$

where $\left\|f^{\prime \prime}\right\|=\sup _{x \in(a, b)}\left|f^{\prime \prime}(x)\right|$.

Proof. From the right hand side of the inequality (2.7), we have

$$
\begin{aligned}
\frac{(b-a)^{2}}{24 n^{2}} A\left(z_{1}, z_{2}, \ldots, z_{n}\right) & =\frac{(b-a)^{2}}{24 n^{3}} \sum_{k=1}^{n}\left(\left|f^{\prime \prime}\left(\xi_{k-1}\right)\right|+\left|f^{\prime \prime}\left(\xi_{k}\right)\right|\right) \\
& \leqslant \frac{(b-a)^{2}}{24 n^{3}}\left(2 n\left\|f^{\prime \prime}\right\|\right)=\frac{(b-a)^{2}}{12 n^{2}}\left\|f^{\prime \prime}\right\|
\end{aligned}
$$

and since

$$
\frac{(b-a)^{2}}{12 n^{2}}\left\|f^{\prime \prime}\right\| \leqslant \varepsilon
$$

then for $n$ we get

$$
n \geqslant\left\|\frac{b-a}{2} \sqrt{\frac{\left\|f^{\prime \prime}\right\|}{3 \varepsilon} \|}\right\|
$$

The proof is complete.

Remark 2. Let $R(f, h)$ be the estimation error of the Trapezoidal rule of numerical integration, then by (2.7) we obtain the estimate for the error known in the literature:

$$
R(f, h)=\frac{b-a}{12} h^{2}\left\|f^{\prime \prime}\right\| .
$$

Remark 3. If $\left\|f^{\prime \prime}\right\|=\sup _{x \in[a, b)}\left|f^{\prime \prime}(x)\right|$, then from inequality $(2.8)$, it follows that

$$
\left|\frac{f(a)+f(b)}{2}-\frac{1}{b-a} \int_{a}^{b} f(x) d x\right| \leqslant \frac{(b-a)^{2}}{12}\left\|f^{\prime \prime}\right\|
$$

and from (2.7), by taken into account inequality (2.10), we get

$$
\left|A\left(y_{1}, y_{2}, \ldots, y_{n}\right)-\frac{1}{b-a} \int_{a}^{b} f(x) d x\right| \leqslant \frac{(b-a)^{2}}{12 n^{2}}\left\|f^{\prime \prime}\right\| .
$$

These two inequalities show that when the interval is divided into $n$ sub-intervals, the error of an Hadamard-type inequality decreases in $n^{2}$ times.

Theorem 2.3. Let $f: I \subset \mathbb{R} \rightarrow \mathbb{R}$ be a twice differentiable function on $I^{\circ}$. If $f^{\prime \prime} \in L[a, b]$, where $a, b \in I$ and $\left|f^{\prime \prime}\right|^{q}$ is a convex function on $[a, b]$, then for all $\alpha>2, q>1$, and $\frac{1}{p}+\frac{1}{q}=1$ the following inequality holds

$$
\left|\sum_{k=1}^{n} \frac{f\left(\xi_{k-1}\right)+f\left(\xi_{k}\right)}{2}-\frac{\Gamma(\alpha)}{2 h^{\alpha-2}} \sum_{k=1}^{n} U_{k}\right| \leqslant \frac{h^{2} \varpi}{2 \alpha} \sum_{k=1}^{n} D_{k},
$$


where

$$
\begin{aligned}
\varpi= & \left(\frac{\Gamma(\alpha+1) \Gamma(q+1)}{\Gamma(\alpha+q+2)}\right)^{\frac{1}{q}}, \\
D_{k}= & \left(\alpha\left|f^{\prime \prime}\left(\xi_{k-1}\right)\right|^{q}+(q+1)\left|f^{\prime \prime}\left(\xi_{k-1}\right)\right|^{q}\right)^{\frac{1}{q}} \\
& +\left((q+1)\left|f^{\prime \prime}\left(\xi_{k-1}\right)\right|^{q}+\alpha\left|f^{\prime \prime}\left(\xi_{k-1}\right)\right|^{q}\right)^{\frac{1}{q}} .
\end{aligned}
$$

Proof. By Lemma 2.1 and the triangle inequality, we obtain

$$
\left|\sum_{k=1}^{n} \frac{f\left(\xi_{k-1}\right)+f\left(\xi_{k}\right)}{2}-\frac{\Gamma(\alpha)}{2 h^{\alpha-2}} \sum_{k=1}^{n} U_{k}\right| \leqslant \frac{(b-a)^{2}}{2}\left(\left|I_{1 k}\right|+\left|I_{2 k}\right|\right) .
$$

By using the Hölder integral inequality 1.3 and since $\left|f^{\prime \prime}\right|^{q}$ is a convex function, we have

$$
\begin{aligned}
\left|I_{1 k}\right| & =\left|\int_{0}^{1} t^{\alpha-1}(1-t) f^{\prime \prime}\left(t \xi_{k-1}+(1-t) \xi_{k}\right) d t\right| \\
& \leqslant \int_{0}^{1} t^{\frac{\alpha-1}{p}} t^{\frac{\alpha-1}{q}}(1-t)\left|f^{\prime \prime}\left(t \xi_{k-1}+(1-t) \xi_{k}\right)\right| d t \\
& \leqslant\left(\int_{0}^{1} t^{\alpha-1} d t\right)^{\frac{1}{p}}\left(\int_{0}^{1} t^{\alpha-1}(1-t)^{q}\left(t\left|f^{\prime \prime}\left(\xi_{k-1}\right)\right|^{q}+(1-t)\left|f^{\prime \prime}\left(\xi_{k}\right)\right|^{q}\right) d t\right)^{\frac{1}{q}} \\
& =\alpha^{-\frac{1}{p}}\left(\left|f^{\prime \prime}\left(\xi_{k-1}\right)\right|^{q} \int_{0}^{1} t^{\alpha}(1-t)^{q} d t+\left|f^{\prime \prime}\left(\xi_{k-1}\right)\right|^{q} \int_{0}^{1} t^{\alpha-1}(1-t)^{q+1} d t\right)^{\frac{1}{q}} \\
& =\alpha^{-\frac{1}{p}}\left(B(\alpha+1, q+1)\left|f^{\prime \prime}\left(\xi_{k-1}\right)\right|^{q}+\left|f^{\prime \prime}\left(\xi_{k}\right)\right|^{q} B(\alpha, q+2)\right),
\end{aligned}
$$

where $B(.,$.$) is the Euler Beta functions.$

By the Euler functions properties

$$
\begin{aligned}
B(\alpha+1, q+1) & =\frac{\Gamma(\alpha+1) \Gamma(q+1)}{\Gamma(\alpha+q+2)}=\frac{\alpha \Gamma(\alpha) \Gamma(q+1)}{\Gamma(\alpha+q+2)}, \\
B(\alpha, q+2) & =\frac{\Gamma(\alpha) \Gamma(q+2)}{\Gamma(\alpha+q+2)}=\frac{(q+1) \Gamma(\alpha) \Gamma(q+1)}{\Gamma(\alpha+q+2)},
\end{aligned}
$$

for the first integral, we get

$$
\begin{aligned}
\left|I_{1 k}\right| & \leqslant\left(\frac{1}{\alpha}\right)^{1-\frac{1}{q}}\left(\frac{\Gamma(\alpha) \Gamma(q+1)}{\Gamma(\alpha+q+2)}\right)^{\frac{1}{q}}\left(\alpha\left|f^{\prime \prime}\left(\xi_{k-1}\right)\right|^{q}+(q+1)\left|f^{\prime \prime}\left(\xi_{k-1}\right)\right|^{q}\right)^{\frac{1}{q}} \\
& =\frac{1}{\alpha}\left(\frac{\Gamma(\alpha+1) \Gamma(q+1)}{\Gamma(\alpha+q+2)}\right)^{\frac{1}{q}}\left(\alpha\left|f^{\prime \prime}\left(\xi_{k-1}\right)\right|^{q}+(q+1)\left|f^{\prime \prime}\left(\xi_{k-1}\right)\right|^{q}\right)^{\frac{1}{q}}\left|I_{1 k}\right| \\
& \leqslant \frac{\varpi}{\alpha} \cdot\left(\alpha\left|f^{\prime \prime}\left(\xi_{k-1}\right)\right|^{q}+(q+1)\left|f^{\prime \prime}\left(\xi_{k-1}\right)\right|^{q}\right)^{\frac{1}{q}} .
\end{aligned}
$$

Similarly, for the second integral $I_{2 k}$, we can write

$$
\left|I_{2 k}\right| \leqslant \frac{\varpi}{\alpha} \cdot\left((q+1)\left|f^{\prime \prime}\left(\xi_{k-1}\right)\right|^{q}+\alpha\left|f^{\prime \prime}\left(\xi_{k-1}\right)\right|^{q}\right)^{\frac{1}{q}} .
$$

By adding the last inequalities, we get

$$
\left|I_{1 k}\right|+\left|I_{2 k}\right| \leqslant \frac{\varpi}{\alpha} \cdot D_{k}
$$


By multiplying both sides of inequality 2.15 by the expression $\frac{h^{2}}{2}$ and taking into account (2.14), we obtain inequality (2.13). The proof is complete.

Corollary 3. If $\alpha=2$, then from (2.13), we get

$$
\left|A\left(y_{1}, y_{2}, \ldots, y_{n}\right)-\frac{1}{b-a} \int_{a}^{b} f(x) d x\right| \leqslant \frac{(b-a)^{2} \varpi(q)}{8 n^{2}} A\left(D_{1}, D_{2}, \ldots, D_{n}\right),
$$

where

$$
\begin{aligned}
\varpi(q) & =\left(\frac{2}{(q+1)(q+2)(q+3)}\right)^{\frac{1}{q}}, \\
D_{k} & =\left(2\left|f^{\prime \prime}\left(\xi_{k-1}\right)\right|^{q}+(q+1)\left|f^{\prime \prime}\left(\xi_{k}\right)\right|^{q}\right)^{\frac{1}{q}}+\left((q+1)\left|f^{\prime \prime}\left(\xi_{k-1}\right)\right|^{q}+2\left|f^{\prime \prime}\left(\xi_{k}\right)\right|^{q}\right)^{\frac{1}{q}} .
\end{aligned}
$$

Proof. For $\alpha=2$ as shown in Corollary 1, the left hand side of the inequality (2.13) will be

$$
\left|\sum_{k=1}^{n} \frac{f\left(\xi_{k-1}\right)+f\left(\xi_{k}\right)}{2}-\frac{\Gamma(\alpha)}{2 h^{\alpha-2}} \sum_{k=1}^{n} U_{k}\right|=\left|\sum_{k=1}^{n}\left(f\left(\xi_{k}\right)+f\left(\xi_{k-1}\right)\right)-\frac{2 n}{b-a} \int_{a}^{b} f(x) d x\right| .
$$

For the right hand side of inequality (2.13), we get

$$
\begin{aligned}
\varpi(q) & =\left(\frac{\Gamma(\alpha+1) \Gamma(q+1)}{\Gamma(\alpha+q+2)}\right)^{\frac{1}{q}}=\left(\frac{\Gamma(3) \Gamma(q+1)}{\Gamma(q+4)}\right)^{\frac{1}{q}} \\
& =\left(\frac{2 q \Gamma(q)}{(q+3)(q+2)(q+1) q \Gamma(q)}\right)^{\frac{1}{q}}=\left(\frac{2}{(q+3)(q+2)(q+1)}\right)^{\frac{1}{q}} .
\end{aligned}
$$

Hence, inequality (2.13) becomes

$$
2 n\left|\frac{1}{n} \sum_{k=1}^{n}\left(\frac{f\left(\xi_{k}\right)+f\left(\xi_{k-1}\right)}{2}\right)-\frac{1}{b-a} \int_{a}^{b} f(x) d x\right| \leqslant \frac{h^{2} \varpi(q)}{4} \sum_{k=1}^{n} D_{k} .
$$

By dividing both sides of the last inequality by $2 n$, we get (2.16).

Remark 4. For $n=1$, by 2.16) we get

$$
\left|\frac{f(a)+f(b)}{2}-\frac{1}{b-a} \int_{a}^{b} f(x) d x\right| \leqslant \frac{(b-a)^{2}}{8} \varpi(q) \cdot D_{1},
$$

where

$$
\begin{aligned}
\varpi(q) & =\left(\frac{2}{(q+1)(q+2)(q+3)}\right)^{\frac{1}{q}}, \\
D_{1} & =\left(2\left|f^{\prime \prime}(a)\right|^{q}+(q+1)\left|f^{\prime \prime}(b)\right|^{q}\right)^{\frac{1}{q}}+\left((q+1)\left|f^{\prime \prime}(a)\right|^{q}+2\left|f^{\prime \prime}(b)\right|^{q}\right)^{\frac{1}{q}} .
\end{aligned}
$$

Since the

$$
\lim _{q \rightarrow 1^{+}}\left(\frac{2}{(q+1)(q+2)(q+3)}\right)^{\frac{1}{q}}=\frac{1}{12}
$$

and

$$
\lim _{q \rightarrow \infty}\left(\frac{2}{(q+1)(q+2)(q+3)}\right)^{\frac{1}{q}}=1,
$$


we hence have

$$
\frac{1}{12} \leqslant\left(\frac{3}{(q+1)(q+2)(q+3)}\right)^{\frac{1}{q}}<1
$$

for all $q>1$. For $q \rightarrow 1^{+}$, by (2.18) we get (2.8).

Theorem 2.4. Let $f: I \subset \mathbb{R} \rightarrow \mathbb{R}$ be a twice differentiable function on $I^{\circ}$. If $f^{\prime \prime} \in L[a, b]$, where $a, b \in I$ and $\left|f^{\prime \prime}\right|^{q}$ is a convex function on $[a, b]$, then for all $\alpha>2$ and $q \geqslant 1$ the following inequality holds

$$
\left|\sum_{k=1}^{n} \frac{f\left(\xi_{k-1}\right)+f\left(\xi_{k}\right)}{2}-\frac{\Gamma(\alpha)}{2 h^{\alpha-2}} \sum_{k=1}^{n} U_{k}\right| \leqslant \frac{h^{2} \vartheta}{2} \sum_{k=1}^{n} V_{k},
$$

where

$$
\begin{aligned}
\vartheta & =\frac{1}{\alpha(\alpha+1)}\left(\frac{\alpha}{\alpha+2}\right)^{\frac{1}{q}} \\
V_{k} & =\left(\left|f^{\prime \prime}\left(\xi_{k-1}\right)\right|^{q}+\frac{2}{\alpha}\left|f^{\prime \prime}\left(\xi_{k}\right)\right|^{q}\right)^{\frac{1}{q}}+\left(\frac{2}{\alpha}\left|f^{\prime \prime}\left(\xi_{k-1}\right)\right|^{q}+\left|f^{\prime \prime}\left(\xi_{k}\right)\right|^{q}\right)^{\frac{1}{q}} .
\end{aligned}
$$

Proof. Owing to identity 2.2 and the triangle inequality, we can write:

$$
\left|\sum_{k=1}^{n} \frac{f\left(\xi_{k-1}\right)+f\left(\xi_{k}\right)}{2}-\frac{\Gamma(\alpha)}{2 h^{\alpha-2}} \sum_{k=1}^{n} U_{k}\right| \leqslant \frac{(b-a)^{2}}{2}\left(\left|I_{1 k}\right|+\left|I_{2 k}\right|\right) .
$$

By using the well-known power mean integral inequality (1.4) and since $\left|f^{\prime \prime}\right|^{q}$ is a convex function, we have

$$
\begin{aligned}
\left|I_{1 k}\right|= & \left|\int_{0}^{1} t^{\alpha-1}(1-t) f^{\prime \prime}\left(t \xi_{k-1}+(1-t) \xi_{k}\right) d t\right| \leqslant\left(\int_{0}^{1} t^{\alpha-1}(1-t) d t\right)^{1-\frac{1}{q}} \\
& \cdot\left(\int_{0}^{1} t^{\alpha-1}(1-t)\left(t\left|f^{\prime \prime}\left(\xi_{k-1}\right)\right|^{q}+(1-t)\left|f^{\prime \prime}\left(\xi_{k}\right)\right|^{q}\right) d t\right)^{\frac{1}{q}} \\
= & \left(\frac{1}{\alpha(\alpha+1)}\right)^{1-\frac{1}{q}}\left(\left|f^{\prime \prime}\left(\xi_{k-1}\right)\right|^{q} \int_{0}^{1}(1-t) t^{\alpha} d t+\left|f^{\prime \prime}\left(\xi_{k}\right)\right|^{q} \int_{0}^{1} t^{\alpha-1}(1-t)^{2} d t^{\frac{1}{q}}\right) \\
= & \left(\frac{1}{\alpha(\alpha+1)}\right)^{1-\frac{1}{q}}\left(\frac{1}{(\alpha+1)(\alpha+2)}\left|f^{\prime \prime}\left(\xi_{k-1}\right)\right|^{q}+\frac{2}{\alpha(\alpha+1)(\alpha+2)}\left|f^{\prime \prime}\left(\xi_{k}\right)\right|^{q}\right)^{\frac{1}{q}} \\
= & \left(\frac{1}{\alpha(\alpha+1)}\right)^{1-\frac{1}{q}}\left(\frac{1}{(\alpha+1)(\alpha+2)}\right)^{\frac{1}{q}}\left(\left|f^{\prime \prime}\left(\xi_{k-1}\right)\right|^{q}+\frac{2}{\alpha}\left|f^{\prime \prime}\left(\xi_{k}\right)\right|^{q}\right)^{\frac{1}{q}}
\end{aligned}
$$

or

$$
\left|I_{1 k}\right| \leqslant \frac{1}{\alpha(\alpha+1)}\left(\frac{\alpha}{\alpha+2}\right)^{\frac{1}{q}}\left(\left|f^{\prime \prime}\left(\xi_{k-1}\right)\right|^{q}+\frac{2}{\alpha}\left|f^{\prime \prime}\left(\xi_{k}\right)\right|^{q}\right)^{\frac{1}{q}} .
$$

Similarly, for the second integral, we can write

$$
\left|I_{2 k}\right| \leqslant \frac{1}{\alpha(\alpha+1)}\left(\frac{\alpha}{\alpha+2}\right)^{\frac{1}{q}}\left(\frac{2}{\alpha}\left|f^{\prime \prime}\left(\xi_{k-1}\right)\right|^{q}+\left|f^{\prime \prime}\left(\xi_{k}\right)\right|^{q}\right)^{\frac{1}{q}} .
$$


By adding side by side the last inequalites $(2.21)$ and $(2.22)$, we get

$$
\left|I_{1 k}\right|+\left|I_{2 k}\right| \leqslant \frac{1}{\alpha(\alpha+1)}\left(\frac{\alpha}{\alpha+2}\right)^{\frac{1}{q}} V_{k} .
$$

By multiplying both sides of inequality 2.23 by the expression $\frac{h^{2}}{2}$ and taking into account (2.20), we obtain inequality 2.19). The proof is completed.

Corollary 4. For $\alpha=2$, from Theorem 2.4, we get

$$
\left|A\left(y_{1}, y_{2}, \ldots, y_{n}\right)-\frac{1}{b-a} \int_{a}^{b} f(x) d x\right| \leqslant \frac{(b-a)^{2}}{12 n^{2}} A\left(v_{1}, v_{2}, \ldots, v_{n}\right),
$$

where

$$
v_{k}=\left(\frac{\left|f^{\prime \prime}\left(\xi_{k-1}\right)\right|^{q}+\left|f^{\prime \prime}\left(\xi_{k}\right)\right|^{q}}{2}\right)^{\frac{1}{q}}, k=1,2, \ldots, n .
$$

Proof. If we take $\alpha=2$, then from Theorem 2.4, we have

$$
\begin{aligned}
\vartheta & =\frac{1}{\alpha(\alpha+1)}\left(\frac{\alpha}{\alpha+2}\right)^{\frac{1}{q}}=\frac{1}{6}\left(\frac{1}{2}\right)^{\frac{1}{q}} \\
V_{k} & =\left(\left|f^{\prime \prime}\left(\xi_{k-1}\right)\right|^{q}+\frac{2}{\alpha}\left|f^{\prime \prime}\left(\xi_{k}\right)\right|^{q}\right)^{\frac{1}{q}}+\left(\frac{2}{\alpha}\left|f^{\prime \prime}\left(\xi_{k-1}\right)\right|^{q}+\left|f^{\prime \prime}\left(\xi_{k}\right)\right|^{q}\right)^{\frac{1}{q}} \\
& =2\left(\left|f^{\prime \prime}\left(\xi_{k-1}\right)\right|^{q}+\left|f^{\prime \prime}\left(\xi_{k}\right)\right|^{q}\right)^{\frac{1}{q}}
\end{aligned}
$$

and, in view of (2.17), we can write

$$
\left|\sum_{k=1}^{n}\left(f\left(\xi_{k}\right)+f\left(\xi_{k-1}\right)\right)-\frac{2 n}{b-a} \int_{a}^{b} f(x) d x\right| \leqslant \frac{(b-a)^{2}}{12 n^{2}}\left(\frac{1}{2}\right)^{\frac{1}{q}} \sum_{k=1}^{n} V_{k}=\frac{(b-a)^{2}}{6 n^{2}} \sum_{k=1}^{n} v_{k} .
$$

By dividing both sides of the last inequality by $2 n$, we get (2.24).

Remark 5. If we choose $n=1$, from (2.24), we get

$$
\left|\frac{f(a)+f(b)}{2}-\frac{1}{b-a} \int_{a}^{b} f(x) d x\right| \leqslant \frac{(b-a)^{2}}{12}\left(\frac{\left|f^{\prime \prime}(a)\right|^{q}+\left|f^{\prime \prime}(b)\right|^{q}}{2}\right)^{\frac{1}{q}} .
$$

For $q=1$, from 2.25), we get 2.8.

\section{BIBLIOGRAPHY}

1. S.S. Dragomir, C.E.M. Pearce. Selected topics on Hermite - Hadamard inequalities and applications. RGMIA Monographs, Victoria University (2000).

2. J. Hadamard. Étude sur les propriétés des fonctions entières en particulier d'une fonction considéréepar Riemann // J. Math. Ser. 4. IX, 171-215 (1893).

3. S. Miller and B. Ross. An introduction to the fractional calculus and fractional differential equations. John Wiley \& Sons, New York (1993).

4. A.M. Nakhushev. Fractional calculus and its application. Fizmatlit, Moscow (2003) (in Russian).

5. A.G. Butkovskii, S.S. Postnov, E.A. Postnova. Fractional integro-differential calculus and its control-theoretical applications. I. Mathematical fundamentals and the problem of interpretation // Avtomat. Telemekh. 4:3, 3-42 (2013). [Autom. Remote Control. 74:4, 543-574 (2013).]

6. P.O. Mohammed, F.K. Hamasalh. New conformable fractional integral inequalities of HermiteHadamard type for convex functions // Symmetry. 11:2, 263 (2019). 
7. J.E. Napoles Valdes, J. M Rodriguez, J.M. Sigarreta. New Hermite-Hadamard type inequalities involving non-conformable integral operators // Symmetry 11:9, 1108 (2019).

8. D.S. Mitrinovic, J. Peĉariĉ, A.M. Fink. Classical and new inequalities in analysis. Kluwer Academic, Dordrecth (1993).

9. M.Z. Sarıkaya, E. Set, H. Yaldiz and N. Bashak. Hermite-Hadamard's inequalities for fractional integrals and related fractional inequalities // Math. Comp. Model. 57, 2403-2407 (2013).

10. B. Bayraktar. Some new inequalities of Hermite-Hadamard type for differentiable GodunovaLevin functions via fractional integrals // Konuralp J. Math. 8:1, 91-96 (2020).

11. B. Bayraktar. Some integral inequalities of Hermite-Hadamard type for differentiable $(s, m)-$ convex functions via fractional integrals // TWMS J. App. Eng. Math. 10:3, 625-637 (2020).

12. B. Bayraktar. Some new generalizations of Hadamard-type Midpoint inequalities involving fractional integrals // Probl. Anal. Issues Anal. 9:27-3, 66-82 (2020).

13. B. Bayraktar. Some integral inequalities for functions whose absolute values of the third derivative is concave and $r$-convex // Turkish J. Ineq. 4:2, 59-78 (2020).

14. M.A. Latif, S.S. Dragomir. New inequalities of Hermite-Hadamard type for functions whose derivatives in absolute value are convex with applications// Acta Univ. M. Belii, Ser. Math. 21, $27-42(2013)$.

15. M. E. Özdemir, A. Ekinci, A.O. Akdemir. Some new integral inequalities for functions whose derivatives of absolute values are convex and concave // TWMS J. Pure Appl. Math. 10:2, 212-224 (2019).

16. A. Ekinci, M. E. Özdemir. Some new integral inequalities via Riemann-Liouville integral operators // Appl. Comput. Math. 18:3, 288-295 (2019).

17. O. Almutairi and A. Kılıçman. Generalized integral inequalities for Hermite-Hadamard-type inequalities via s-convexity on fractal sets // Mathematics. 7:11, 1065 (2019).

18. M.Z. Sarıkaya, N. Aktan. On the generalization of some integral inequalities and their applications // Math. Comput. Model. 54, 2175-2182 (2011).

Bahtiyar Bayraktar,

Bursa Uludag University,

Faculty of Education,

Gorukle Campus,

16059, Bursa, Turkey

E-mail: bbayraktar@uludag.edu.tr

Muhammet Emin Özdemir,

Bursa Uludag University,

Faculty of Education,

Gorukle Campus,

16059, Bursa, Turkey

E-mail: eminozdemir@uludag.edu.tr 